\title{
Post-infective and chronic fatigue syndromes precipitated by viral and non-viral pathogens: prospective cohort study
}

Ian Hickie, Tracey Davenport, Denis Wakefield, Ute Vollmer-Conna, Barbara Cameron, Suzanne D Vernon, William C Reeves, Andrew Lloyd, for the Dubbo Infection Outcomes Study Group

\begin{abstract}
Objective To delineate the risk factors, symptom patterns, and longitudinal course of prolonged illnesses after a variety of acute infections.

Design Prospective cohort study following patients from the time of acute infection with Epstein-Barr virus (glandular fever), Coxiella burnetii ( $\mathrm{Q}$ fever), or Ross River virus (epidemic polyarthritis).

Setting The region surrounding the township of Dubbo in rural Australia, encompassing a $200 \mathrm{~km}$ geographical radius and 104400 residents.

Participants 253 patients enrolled and followed at regular intervals over 12 months by self report, structured interview, and clinical assessment.

Outcome measures Detailed medical, psychiatric, and laboratory evaluations at six months to apply diagnostic criteria for chronic fatigue syndrome. Premorbid and intercurrent illness characteristics recorded to define risk factors for chronic fatigue syndrome. Self reported illness phenotypes compared between infective groups.

Results Prolonged illness characterised by disabling fatigue, musculoskeletal pain, neurocognitive difficulties, and mood disturbance was evident in 29 (12\%) of 253 participants at six months, of whom 28 (11\%) met the diagnostic criteria for chronic fatigue syndrome. This post-infective fatigue syndrome phenotype was stereotyped and occurred at a similar incidence after each infection. The syndrome was predicted largely by the severity of the acute illness rather than by demographic, psychological, or microbiological factors.

Conclusions A relatively uniform post-infective fatigue syndrome persists in a significant minority of patients for six months or more after clinical infection with several different viral and non-viral micro-organisms. Post-infective fatigue syndrome is a valid illness model for investigating one pathophysiological pathway to chronic fatigue syndrome.
\end{abstract}

\section{Introduction}

Chronic fatigue syndrome is defined as persistent or relapsing fatigue that cannot be explained by other medical or psychiatric conditions, which has been present for at least six months, is not alleviated by rest, and causes substantial reduction in daily activities. ${ }^{1}$ Although chronic fatigue syndrome is commonly reported to develop after an acute infective illness, ${ }^{2}{ }^{3}$ many case-control studies have failed to find consistent associations between chronic fatigue syndrome and either known or novel infectious agents. ${ }^{4-6}$ Post-infective fatigue states have a long history and have been linked to a diverse spectrum of infections, including brucellosis (which is caused by an intracellular bacterium), ${ }^{7}$ glandular fever (caused by the herpesvirus Epstein-Barr virus), ${ }^{8}$ Lyme disease (caused by infection with the tickborne spirochaete Borrelia burgdorferi), ${ }^{9} \mathrm{Q}$ fever (caused by the intracellular, rickettsia-like pathogen Coxiella burnetii), ${ }^{10}$ Ross River virus (a mosquito-borne arbovirus found in countries around the Pacific rim), ${ }^{11}$ and viral meningitis (most commonly caused by enteroviral infection). ${ }^{12}$ By contrast, a comprehensive prospective study of clinical outcomes after other common, more minor, viral infections found no association with prolonged fatigue. ${ }^{13}$ Population based prospective studies of the spectrum of post-infective fatigue states are therefore needed to delineate the key symptoms and longitudinal course of the post-infective fatigue syndrome; to identify demographic, microbial, immunological, and psychological risk factors; and to determine whether disparate pathogens can precipitate chronic fatigue syndrome.

\section{Methods}

\section{Study site}

The ongoing Dubbo infection outcomes study is centred on the township of Dubbo in a rural region of southwestern Australia, encompassing a $200 \mathrm{~km}$ radius and 104400 residents (Australian Bureau of Statistics, 2001). The population includes approximately 8\% Aboriginal Australians.

\section{Participants}

The 94 family practitioners and all four diagnostic pathology laboratories that serve the region cooperated to provide us with coded reports of all IgM positive serological results indicating acute Epstein-Barr virus, Q fever, or Ross River virus infections. Patients aged 16 years or over, who provided written informed consent, were enrolled through their family doctor. We excluded patients who had symptoms present for more than six weeks or reported pre-existing medical disorders or drug use likely to be associated with prolonged fatigue. After the baseline assessment, we followed up participants at three weeks, six weeks, and three months, after which we further evaluated a matched case-control series (see below). We reviewed all enrolled participants again at 12 months.

\section{Interview schedules and self report instruments}

At enrolment, the study nurse recorded the clinical, medical, psychiatric, and family history. A semi-structured psychiatric 


\begin{tabular}{|c|c|c|c|c|c|}
\hline Serology & No & Mean (range) age (years) & Female & Education* & Employed† \\
\hline EBV confirmed $\ddagger$ & 68 & $22(16-49)$ & $39 / 68$ (57) & $15 / 64(23)$ & $30 / 66$ (45) \\
\hline RRV confirmed $\ddagger$ & 60 & $40(18-69)$ & $27 / 60(45)$ & $25 / 58$ (43) & $47 / 58$ (81) \\
\hline Q fever confirmed $\neq$ & 43 & $40(16-73)$ & $6 / 43(14)$ & $21 / 41 \quad(51)$ & $40 / 42(95)$ \\
\hline Not confirmed§ & 82 & $38(16-77)$ & $36 / 82$ (44) & $34 / 77$ (44) & $60 / 79(76)$ \\
\hline All subjects & 253 & $34(16-77)$ & $108 / 253 \quad(43)$ & $95 / 240(40)$ & $177 / 245(72)$ \\
\hline
\end{tabular}

EBV=Epstein-Barr virus; RRV=Ross River virus.

${ }^{*}$ Completed 10 years or less of formal education.

†Currently in paid employment.

$\ddagger$ Acute infection confirmed by testing of longitudinally collected sera.

§Provisional diagnosis of EBV, RRV, or $Q$ fever infection not confirmed by repeated testing of longitudinally collected sera.

interview (composite international diagnostic interview; CIDI) was used to record premorbid and intercurrent psychiatric disorders. ${ }^{14}$ At each visit, we recorded detailed self report assessments of physical and psychological health and collected a blood sample. We used the 34 item somatic and psychological health report (SPHERE) to monitor a wide range of physical and psychological symptoms..$^{15}$ An empirically derived subscale that records somatic symptoms (the SOMA) identified the key clinical features of prolonged fatigue states. ${ }^{16}$ The temporal stability and construct validity of this instrument have been shown. ${ }^{16} \mathrm{~A}$ threshold score of 3 (out of the possible 12) on this subscale predicts disability ratings, as well as both patients' and doctors' reports of reasons for presentation to primary care. ${ }^{16}$ Participants also completed the brief disability questionnaire to document functional impairment, ${ }^{18}$ a shortened Eysenck personality inventory-neuroticism scale,${ }^{19}$ and the locus of control of behaviour scale ${ }^{20}$ to measure these risk factors for anxiety and depression.

\section{Case definitions}

We classified participants as provisional cases of post-infective fatigue syndrome if their SOMA scores at all time points up to and including three months exceeded the established threshold score. ${ }^{16}$ We invited these cases, and control participants matched by age and sex who had recovered promptly from the same infection, at six months for a medical interview, examination by a physician (AL), and laboratory investigation to exclude alternative medical explanations for ongoing symptoms, such as hypothyroidism or primary sleep disorder. A psychiatrist (IH) also assessed them, to ensure that no exclusionary psychiatric diagnosis was evident and to allocate comorbid diagnoses according to the Diagnostic and Statistical Manual of Mental Disorders, fourth edition (DSM-IV). Where appropriate, AL and IH diagnosed the chronic fatigue syndrome (termed here confirmed post-infective fatigue syndrome) by consensus at six months after the onset of symptoms, according to the international diagnostic criteria.

\section{Laboratory investigations}

We confirmed the initial serological diagnoses by testing acute and convalescent sera. Diagnosis of acute Epstein-Barr virus infection required seroconversion to IgG anti-Epstein-Barr virus viral capsid antigen or detection of IgM anti-viral capsid antigen and low avidity IgG anti-viral capsid antigen in the baseline sample. ${ }^{21}$ We confirmed diagnoses of acute Ross River virus infection by demonstration of IgG seroconversion or by detection of IgM anti-Ross River virus and low baseline avidity IgG anti-Ross River virus (Lloyd A et al, submitted for publication). We confirmed primary $Q$ fever infections by detection of IgG seroconversion or detection of IgM antibodies and a fourfold rise in antibody titre in immunofluorescence assays using $C$ burnetii phase I and phase II antigens. ${ }^{22}$

\section{Statistical analysis}

We used SPSS 12.0.1 for statistical analyses. To describe the clinical phenotypes (both cross sectionally and longitudinally) and to assign values to the severity of each symptom domain, we did factor analysis with principal axis factoring and varimax rotation; we included all 34 items of the somatic and psychological health report from the 229 participants who had full data available at assessments done four to eight weeks after the onset of acute illness. For calculation of the case rates for post-infective fatigue syndrome at each time point, we designated participants who discontinued $(n=26)$ as recovered and retained them in the denominator. We used Spearman rank order correlations to assess associations between symptom factor scores and disability measures. We assessed risk factors for caseness at each time point with stepwise multiple regression analyses. To understand the patterns of resolution of the symptom domains, we calculated gradients of the change in mean factor scores per unit of time, and we did planned contrast analyses to compare these data across the infective subgroups of participants.

\section{Results}

\section{Demographic characteristics}

We received laboratory notifications of 855 potential participants with IgM positive results over a five year study period. We were able to contact 430 of these through their family doctor, and $253(59 \%)$ of them agreed to detailed longitudinal assessments. The demographic and illness characteristics of these 253 participants (summarised in table 1) were not significantly different from an additional 177 who agreed to be followed by self report only-age 34.3 versus 37.0 years, difference $=-2.7$ years $(95 \%$ confidence interval -6.08 to 0.67$)$ years; sex (per cent male) $57 \%$ versus $56 \%$, difference $=2 \%$ $(-10 \%$ to $13 \%)$. We found a non-significant trend towards higher baseline symptom scores and worsened disability parameters in the self report cohort compared with the main cohort. These groups of patients did not differ from those who declined enrolment (data not shown).

The demographic features of the main cohort were consistent with the expected patterns of exposure to these pathogens; Epstein-Barr virus infection was most common in adolescents and young adults, $Q$ fever was most common in men (largely because of the nature of the occupational exposure, such as meat working or shearing), and Ross River virus was most common in participants with outdoor activities that increase the likelihood of mosquito bites. ${ }^{23-25}$ In all three infection groups, approximately $25 \%$ of the original serological diagnoses were not confirmed by our more stringent criteria applied longitudinally. This is consistent with the recognised limitations of diagnoses made on the basis of detection of IgM antibodies in a single serum sample. 


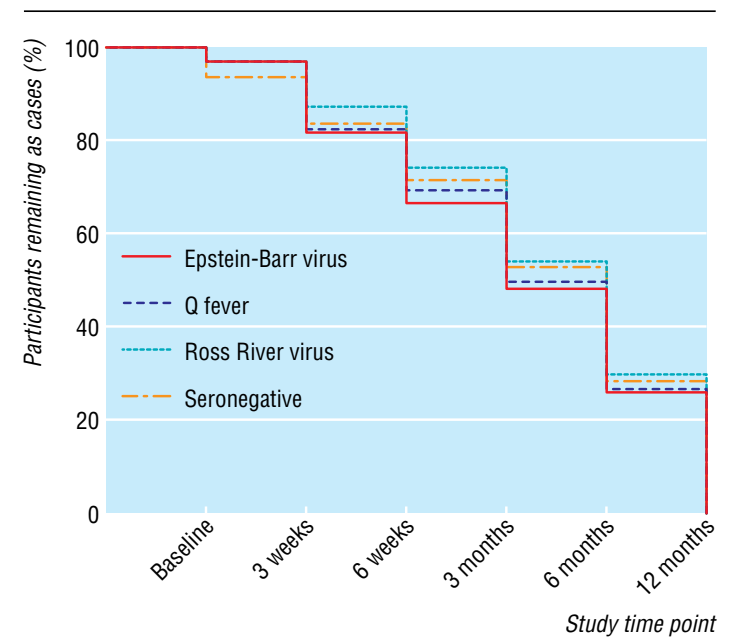

Fig 1 Survival curves of post-infective fatigue syndrome by infective agent after onset of acute infection. Kaplan-Meier analyses of proportion of participants within each infective subcohort who remained as cases. Test of equality across groups: $\chi^{2}=3.45, \mathrm{df}=3, \mathrm{P}>0.05$

\section{Factor analysis of symptoms}

We derived six symptom domains. Two factors seemed to capture the physical and mental distress of acute illness, and we labelled them "acute sickness" (including items such as "headaches" and "fevers") and "irritability" (including "feeling irritable or cranky" and "rapidly changing moods"). We also identified four other factors reminiscent of classic descriptions of post-infective or chronic fatigue states. These included: a "fatigue" factor (with items such as "prolonged tiredness after activity" and "feeling tired after rest or relaxation"), a "musculoskeletal pain" factor (with items such as "pains in your arms or legs" and "joint pain"), a "mood disturbance" factor (with items such as "feeling nervous or tense" and "feeling unhappy or depressed"), and a "neurocognitive disturbance" factor (featuring "poor memory" and "poor concentration"). Scores on the fatigue factor showed the strongest and most consistent correlations with functional impairment: "days out of role in the past month" (baseline $r=0.22, \mathrm{P}<0.01$; three months $r=0.37, \mathrm{P}<0.01$; six months $r=0.25, \mathrm{P}<0.01)$.

\section{Incidence of post-infective fatigue syndrome}

The case rate for provisional post-infective fatigue syndrome was $35 \%(87 / 250)$ at six weeks, $27 \%(67 / 250)$ at three months, $12 \%$ $(29 / 250)$ at six months, and 9\% (22/250) at 12 months. No difference in these case rates existed between the initial infective agents (fig 1).

The medical, psychiatric, and laboratory assessments of the 29 provisional cases of post-infective fatigue syndrome at six months led to exclusion of one participant on medical grounds and none on psychiatric grounds. The 28 cases of chronic fatigue syndrome, termed here confirmed post-infective fatigue syndrome, included 14 men and 14 women with a mean age of 37 (range 17-63) years, including five participants with confirmed Epstein-Barr virus infection, three with Q fever, 13 with Ross River virus, and eight with unconfirmed infection. The 28 cases did not differ in age or sex when compared with either all participants with serological confirmation-age 36.0 versus 32.2 years, difference $=3.8(-3.0$ to 10.6$)$ years; sex (per cent male) $55 \%$ versus $58 \%$, difference $=-3.4 \%(-19 \%$ to $28 \%)$ - or all enrolled participants-age 36.0 versus 34 .1 years, mean difference $=1.9(-8.7$ to 4.9$)$ years; sex (per cent male) $55 \%$ versus $58 \%$, difference $=-3 \%(-20 \%$ to $26 \%)$.
The rates of premorbid psychiatric diagnoses in the confirmed cases of post-infective fatigue syndrome and the matched (recovered) control participants, determined by formal psychiatric assessment of both groups at six months, were comparable $-21 \%$ versus $17 \%$, difference $=5 \% \quad(-18 \%$ to $27 \%$ - as were the rates of intercurrent psychiatric disorders$21 \%$ versus $10 \%$, difference $=11 \%(-10 \%$ to $33 \%)$. Similarly, the rates of psychiatric disorder between cases and all remaining participants, detected by the structured interview at baseline, did not differ-premorbid psychiatric disorder 23\% versus 14\%, difference $=9 \%(-23 \%$ to $13 \%)$; intercurrent psychiatric disorder $23 \%$ versus $10 \%$, difference $=13 \%(-8 \%$ to $28 \%)$. Interestingly, the case rates of provisional post-infective fatigue syndrome in the self report cohort were significantly higher at six and 12 months (35\% and 32\%) than in the main cohort. Higher rates of disability were also reported in the self report cohort.

\section{Characteristics of post-infective fatigue syndrome}

If the same pathophysiology underpinned all the clinical aspects of the acute infective illness and the post-infective fatigue state, we would predict that the individual symptom factors that we had derived empirically would resolve in a uniform manner across the time points assessed. In fact, we found substantial variation, particularly early in the course of the illness. In the group of 28 confirmed cases of post-infective fatigue syndrome, the median score on the acute sickness factor rapidly dropped to zero, whereas the median scores for fatigue, musculoskeletal pain, and neurocognitive disturbance remained high (fig 2). When we compared the kinetics of resolution of the symptom factors for the group as a whole, again the acute sickness and irritability factors showed the greatest initial speed of resolution. By contrast, the fatigue and neurocognitive disturbance factors showed significant reductions only late in the course of the illness (fig 3). These differences were most significant in the period between baseline and three months, when planned contrasts showed that the key construct of fatigue differed from all other factors (all $\mathrm{P}<0.05$ ), with the exception of neurocognitive disturbance. When we compared the gradients between three and six months, significant differences no longer existed, suggesting that the symptom domains had become more uniform and stable over time.

Importantly, these final symptom patterns were also highly stereotyped, regardless of the original infective trigger. Planned contrasts of the patterns of resolution of the six symptom factors by infective subcohorts revealed that only musculoskeletal pain showed significant differences in prevalence and natural history in the early post-infective period (baseline to three months: Ross River virus $v$ Epstein-Barr virus, $\mathrm{P}<0.001$; Ross River virus $v$ not confirmed, $\mathrm{P}<0.01$; Ross River virus $v \mathrm{Q}$ fever, $\mathrm{P}<0.01$ ). The central symptom domains of post-infective fatigue syndrome did not differ between the infection groups at later time points.

\section{Risk factors for acute sickness}

Demographic characteristics did not generally predict the scores on the six symptom factors recorded at baseline (table 2 ). We saw an association between older age and the fatigue score during the acute illness. Serologically confirmed Ross River virus infection was associated with the severity of the musculoskeletal pain factor, consistent with the propensity of this infection to cause arthralgia. Higher neuroticism and external locus of control scores were associated with more severe mood disturbance.

\section{Risk factors for post-infective fatigue syndrome}

The predictors of post-infective fatigue syndrome over the 12 months after acute infection were largely limited to the factor 

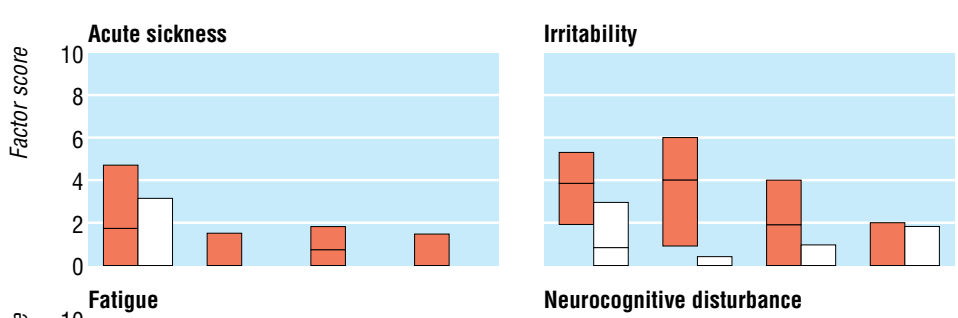

Neurocognitive disturbance
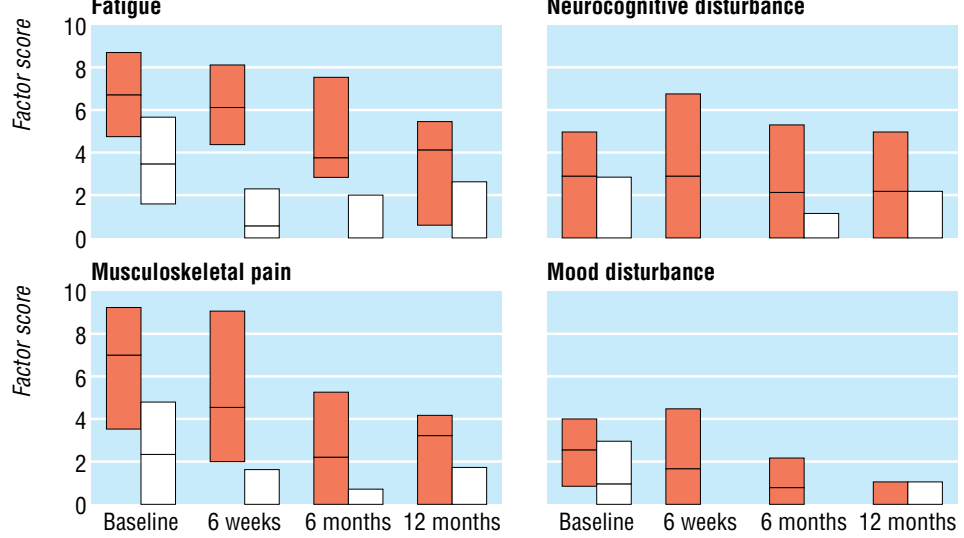

Fig 2 Pattern of change in individual symptom factors in participants with and without post-infective fatigue syndrome. Median (horizontal bar) and 25th/75th centiles (box extremities) of normalised factor scores for six symptom domains in confirmed cases of post-infective fatigue syndrome cases (orange boxes; $\mathrm{n}=28$ ) and those who recovered more promptly (white boxes; $n=225$ )

scores that reflect severity of acute illness (table 3). Importantly, premorbid and intercurrent psychiatric disorder did not show predictive power for post-infective fatigue syndrome at any time point.

\section{Discussion}

Prolonged fatigue states after infections are common and disabling and may persist for 12 months. Although the acute phase of the infections varied, the post-infective fatigue illnesses shared a similar clinical phenotype. Severity of the acute illness, and not demographic or psychological factors, was predictive of post-infective fatigue syndrome.

\section{Strengths and weaknesses}

The application of the chronic fatigue syndrome case definition to designate incident cases in the post-infective setting described

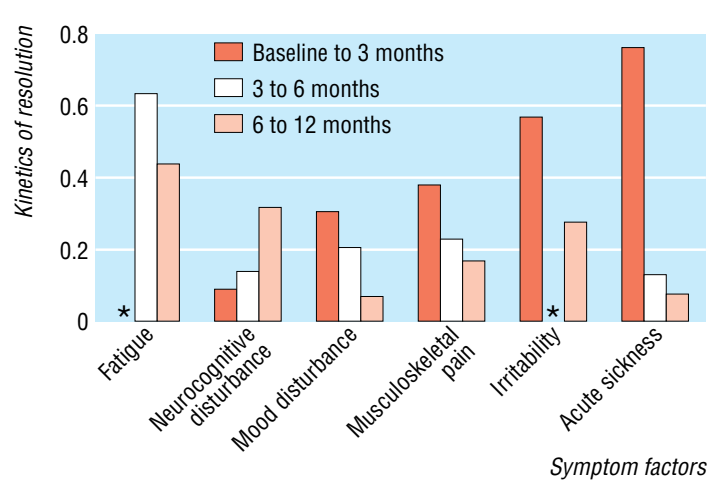

Fig 3 Differential rates of resolution of individual symptom factors after acute infection. Scores on each of six symptom factors for each participant $(n=229)$ over 12 months in three divided time periods, calculated from factor analysis. Mean symptom scores standardised (to ensure comparability) by dividing the mean at each time point for each factor by its mean at baseline. Each bar represents gradient of resolution of factor scores between each time point of assessment. *Period of non-resolution of individual symptom domain between two time points of assessment (that is, gradient $=0$ ) here provides strong evidence for a causative role of these infections in triggering chronic fatigue syndrome. The rate of post-infective fatigue syndrome detected at six months $(11 \%)$ is comparable to those in the three previous cohort studies, which followed only patients with glandular fever. ${ }^{26-31}$ These findings confirm that chronic fatigue syndrome is a relatively common sequel of several different infections-now documented to include Epstein-Barr virus, Ross River virus, and $Q$ fever-but not minor upper respiratory tract or gastrointestinal infections. ${ }^{1}$ Nevertheless, the case rate for post-infective fatigue syndrome in the group who were followed from the serologically unconfirmed infections was similar, suggesting that severity of the acute illness rather than the specific pathogen may be the major determinant of post-infective fatigue syndrome. The patients who elected to participate by self report only with less frequent follow-up apparently had a more severe and protracted illness course, potentially suggesting a bias against inclusion of participants with more severe illness in the main cohort. However, these participants were not evaluated in comparable detail, so these differences may also reflect the higher rates of post-infective fatigue illness and duration in studies based on self report only.

Unlike in previous reports, we required the cases to have serological confirmation of Epstein-Barr virus infection (not required in some studies ${ }^{29}{ }^{30}$ ); continuity of the prolonged fatigue state from onset of the infective illness through to six months (not required in other studies ${ }^{26-31}$ ); and strict application of the diagnostic criteria for chronic fatigue syndrome with exclusion of alternative medical and psychiatric disorders, including through the recommended laboratory investigations ${ }^{1}$ (not required in some studies ${ }^{29}{ }^{30}$ ).

White and colleagues also identified the importance of the specific pathogen and of severity of the acute illness; they found that having a confirmed Epstein-Barr virus infection and severe fatigue at baseline predicted post-infective fatigue syndrome caseness at six months. ${ }^{28}$ In addition, comparable to our findings, 
Research

Table 2 Demographic, psychological, and infective risk factors predicting symptom severity in acute illness $(\mathrm{n}=229)$. Values are standardised $\beta$ coefficients from regression analysis

\begin{tabular}{|c|c|c|c|c|c|c|}
\hline \multirow[b]{2}{*}{ Risk factors } & \multicolumn{6}{|c|}{ Symptom domains $\dagger$} \\
\hline & Acute sickness & Irritability & Musculoskeletal pain & Mood disturbance & $\begin{array}{l}\text { Neurocognitive } \\
\text { disturbance }\end{array}$ & Fatigue \\
\hline \multicolumn{7}{|l|}{ Demographic } \\
\hline Age (years) & 0.04 & 0.03 & 0.004 & -0.15 & -0.09 & $-0.25^{*}$ \\
\hline Sex (female=1) & 0.10 & 0.08 & 0.02 & -0.11 & 0.10 & 0.03 \\
\hline Education (secondary=1) & -0.05 & 0.25 & 0.12 & 0.21 & -0.16 & 0.11 \\
\hline Education (tertiary=1) & 0.08 & 0.24 & 0.16 & 0.18 & -0.03 & 0.11 \\
\hline \multicolumn{7}{|l|}{ Psychological } \\
\hline Premorbid psychiatric disorder (DSM-IV) & -0.10 & 0.06 & -0.05 & -0.03 & -0.19 & -0.05 \\
\hline Intercurrent psychiatric disorder (DSM-IV) & $0.31^{*}$ & -0.07 & 0.07 & 0.14 & $0.43^{* *}$ & -0.12 \\
\hline Neuroticism score & -0.08 & $0.27^{*}$ & $-0.22^{*}$ & $0.45^{\star \star *}$ & 0.03 & 0.16 \\
\hline Locus of control score & $0.27^{\star \star \star}$ & -0.02 & -0.12 & $0.18^{\star}$ & -0.09 & 0.001 \\
\hline \multicolumn{7}{|l|}{ Microbiological } \\
\hline EBV confirmed $\ddagger$ & -0.20 & 0.12 & -0.11 & $-0.26^{*}$ & 0.003 & -0.26 \\
\hline RRV confirmed $\ddagger$ & -0.21 & -0.07 & $0.37^{\star \star *}$ & -0.01 & 0.04 & -0.04 \\
\hline $\bar{Q}$ fever confirmed $\neq$ & -0.03 & -0.01 & -0.003 & -0.10 & 0.08 & 0.05 \\
\hline
\end{tabular}

DSM-IV=Diagnostic and Statistical Manual of Mental Disorders, fourth edition; EBV=Epstein-Barr virus; RRV=Ross River virus.

${ }^{*} \mathrm{P}<0.05$.

${ }^{* *} \mathrm{P}<0.01$.

*** $P<0.001$.

†Derived from initial factor analysis of responses on somatic and psychological health report questionnaire from 229 participants with complete data available at $29-56$ days after onset of

symptoms.

$\ddagger$ Acute infection confirmed by testing of longitudinally collected sera.

premorbid and intercurrent mood disorders were not associated with an increased likelihood of post-infective fatigue syndrome.

A weakness of our study is that the sample size of the participant group reported here did not allow definitive exclusion of risk factors for post-infective fatigue syndrome with small effect sizes. In addition, the participant group enrolled in the cohort was likely to be biased by factors influencing presentation to the general practitioner, including illness severity and psychosocial factors.

Table 3 Risk factors for post-infective fatigue syndrome $(n=229)$. Values are standardised $\beta$ coefficients from regression analysis

\begin{tabular}{|c|c|c|c|}
\hline & 3 months & 6 months $\dagger$ & 12 months \\
\hline \multicolumn{4}{|l|}{ Demographic factors } \\
\hline Age (years) & 0.03 & 0.17 & 0.08 \\
\hline Sex (female=1) & 0.04 & -0.07 & 0.002 \\
\hline Education (secondary=1) & -0.07 & 0.12 & 0.03 \\
\hline Education (tertiary=1) & 0.06 & 0.27 & 0.29 \\
\hline \multicolumn{4}{|l|}{ Baseline symptom factor scores } \\
\hline Acute sickness & 0.06 & -0.11 & -0.002 \\
\hline Irritability & $0.24^{\star}$ & 0.23 & 0.08 \\
\hline Musculoskeletal pain & $0.27^{\star}$ & $0.30^{*}$ & 0.13 \\
\hline Mood disturbance & 0.23 & 0.07 & -0.05 \\
\hline Neurocognitive disturbance & $0.24^{*}$ & 0.20 & 0.14 \\
\hline Fatigue & $0.50^{\star *}$ & $0.35^{\star \star *}$ & $0.27^{*}$ \\
\hline \multicolumn{4}{|l|}{ Psychological factors } \\
\hline Premorbid psychiatric disorder (DSM-IV) & 0.13 & 0.12 & 0.08 \\
\hline Intercurrent psychiatric disorder (DSM-IV) & -0.24 & -0.05 & -0.08 \\
\hline Neuroticism score & 0.04 & 0.07 & 0.20 \\
\hline Locus of control score & -0.004 & 0.17 & 0.11 \\
\hline \multicolumn{4}{|l|}{ Microbiological factors } \\
\hline EBV confirmed & 0.13 & 0.05 & -0.01 \\
\hline RRV confirmed $\ddagger$ & 0.11 & -0.05 & 0.07 \\
\hline $\bar{Q}$ fever confirmed & 0.12 & -0.15 & -0.06 \\
\hline
\end{tabular}

DSM-IV=Diagnostic and Statistical Manual of Mental Disorders, fourth edition;

$E B V=E p s t e i n-B a r r$ virus; $R R V=$ Ross River virus.

${ }^{*} \mathrm{P}<0.05$.

** $\mathrm{P}<0.001$.

†Caseness for chronic fatigue syndrome designated at six months after medical, psychiatric, and laboratory evaluation.

¡Acute infection confirmed by testing of longitudinally collected sera.

\section{Meaning of the study}

Examination of outcomes after the three distinctive acute infections reported here strongly implicates aspects of the host response to infection (rather than the pathogen itself) as the likely determinants of post-infective fatigue syndrome, as the case rates after infection with Epstein-Barr virus (a DNA virus), Ross River virus (an RNA virus), and C burnetii (an intracellular bacterium) were comparable and the symptom characteristics progressively merged over time. In combination with the predominantly self limiting natural history of post-infective fatigue syndrome recorded here, these risk factors and demographic characteristics indicate that patients with postinfective fatigue syndrome constitute a distinguishable subset within the broad diagnostic category of chronic fatigue syndrome. This is consistent with the recognised heterogeneity in patient groups identified within the label of chronic fatigue syndrome. ${ }^{32}$

\section{Unanswered questions and future research}

In patients in this cohort, we recently reported strong, positive correlations with symptoms of acute infection and the spontaneous ex vivo production of the pro-inflammatory cytokines, interleukin $1 \beta$ and interleukin $6 .{ }^{33}$ This finding is consistent with accumulated evidence from animal studies, which indicates that the acute sickness response to infection is mediated by the action of these cytokines on the central nervous system. ${ }^{34}$ However, in the longitudinal study reported here the symptom domains that characterise the acute illness experience (notably "acute sickness" and "irritability") resolved rapidly compared with the domains that are more characteristic of chronic fatigue syndrome ("fatigue" and "neurocognitive disturbance"). Consistent with these data, we have recently found that markers of inflammation and the concentrations of pro-inflammatory cytokines do not remain high in patients with post-infective fatigue syndrome (Vollmer-Conna et al, manuscript in preparation). Similarly, detailed analysis of viral load and antiviral immune responses in a nested case-control series derived from the Epstein-Barr virus cohort did not reveal significant differences between patients with post-infective fatigue syn- 


\section{Research}

drome and those who recovered promptly. ${ }^{35}$ Accordingly, we propose that alternative neurobiological mechanisms triggered during the severe, acute illness and sustained in the absence of ongoing peripheral inflammation underpin the persistent symptom domains of post-infective fatigue syndrome. Further longitudinal case-control studies in the post-infective model will allow testing of this hypothesis.

We gratefully acknowledge the support of the general practitioners and the diagnostic pathology services in the Dubbo region and the enduring cooperation of the participants in the research. Other members of the Dubbo Infection Outcomes Study Group are listed on bmj.com.

Contributors: IH, TD, UV-C, BC, and AL designed and implemented the Dubbo infection outcomes study. SDV and WCR contributed to data analysis and manuscript preparation. All authors commented on and approved the final draft. AL is the guarantor.

Funding: The Dubbo infection outcomes study is funded by project grants from the National Health and Medical Research Council of Australia (No 157092 and No 157062), by Meat \& Livestock, Australia, and by a cooperative research agreement with the Centers for Disease Control and Prevention, USA (No U50/CCU019851-01). The researchers involved in this study were independent of the funding agencies, with the exception of SDV and WCR, who are employees of the Centers for Disease Control, USA.

Competing interests: None declared.

Ethical approval: Human research ethics committees of the University of New South Wales and the Orana and Far West Area Health Service.

1 Fukuda K, Straus SE, Hickie I, Sharpe MC, Dobbins JG, Komaroff A. The chroni fatigue syndrome: a comprehensive approach to its definition and study. Ann Intern Med 1994;121:953-9.

2 Royal Australasian College of Physicians Working Group. Chronic fatigue syndromeclinical practice guidelines 2002. Med J Aust 2002;176:S17-55

3 Afari N, Buchwald D. Chronic fatigue syndrome: a review. Am J Psychiatr 2003;160:221-36

4 Gold D, Bowden R, Sixbey J, Riggs R, Katon WJ, Ashley R, et al. Chronic fatigue: a prospective clinical and virologic study. JAMA 1990;264:48-53.

5 Heneine W, Woods TC, Sinha SD, Khan AS, Chapman LE, Schonberger LB, et al. Lack of evidence for infection with known human and animal retroviruses in patients with chronic fatigue syndrome. Clin Infect Dis 1994;18(suppl 1):S121-5.

6 Mawle AC, Nisenbaum R, Dobbins JG, Gary HE Jr, Stewart JA, Reyes M, et al. Seroepidemiology of chronic fatigue syndrome: a case-control study. Clin Infect Dis $1995 ; 21: 1386-9$.

7 Evans AC. Chronic brucellosis. JAMA 1934;103:665

7 Evans AC. Chronic brucellosis. JAMA 1934;103:665.

(1948:3:858-61.

9 Shadick NA, Phillips CB, Logigian EL, Steere AC, Kaplan RF, Berardi VP, et al. The long-term clinical outcomes of Lyme disease: a population-based retrospective cohort study. Ann Intern Med 1994;121:560-7.

10 Ayres JG, Flint N, Smith EG, Tunnicliffe WS, Fletcher TJ, Hammond K, et al Post-infection fatigue syndrome following Q fever. OJM 1998;91:105-23.

11 Harley D, Bossingham D, Purdie DM, Pandeya N, Sleigh AC. Ross River virus disease in tropical Queensland: evolution of rheumatic manifestations in an inception cohort followed for six months. Med J Aust 2002;177:352-5.

\section{What is already known on this topic}

A post-infective fatigue syndrome that meets diagnostic criteria for chronic fatigue syndrome may follow Epstein-Barr virus infection but not common, minor viral infections

\section{What this study adds}

Post-infective fatigue syndrome represents a common and stereotyped outcome from several viral and non-viral infections

The key risk factor for post-infective fatigue syndrome is the severity of the acute illness and not age, sex, or psychological factors
12 Hotopf M, Noah N, Wessely S. Chronic fatigue and psychiatric morbidity following viral meningitis: a controlled study. J Neurol Neurosurg Psychiatry 1996;60:495-503.

13 Wessely S, Chalder T, Hirsch S, Pawlikowska T, Wallace P, Wright D. Postinfectiou fatigue: prospective cohort study in primary care. Lancet 1995;345:1333-8.

14 Wittchen HU, Robins LN, Cottler LB, Sartorius N, Burke JD, Regier D. Participants in the multicentre WHO/ADAMHA field trials, 1991: cross-cultural feasibility, reliability and sources of variance of the composite international diagnostic interview (CIDI). $B r$ J Psychiatry 1991;159:645-53.

15 Hickie IB, Davenport TA, Hadzi-Pavlovic D, Koschera A, Naismith SL, Scott EM, et al. Development of a simple screening tool for common mental disorders in general practice. Med J Aust 2001:175:S10-7.

16 Hadzi-Pavlovic D, Hickie IB, Wilson AJ, Davenport TA, Lloyd AR. Screening for prolonged fatigue syndromes: validation of the SOFA scale. Soc Psychiatry Psychiatr Epidemiol 2000;35:471-9.

17 Hickie I, Koschera A, Hadzi-Pavlovic D, Bennett B, Lloyd A. The temporal stability and co-morbidity of prolonged fatigue: a longitudinal study in primary care. Psychol Med 1999;29:855-61.

18 Von Korff M, Ustun TB, Ormel J, Kaplan I, Simon GE. Self-report disability in an international primary care study of psychological illness. J Clin Epidemiol 1996;49:297-303.

9 Eysenck HJ, Eysenck SB. Manual of the Eysenck personality questionnaire. London: Hodder and Stoughton, 1975.

20 Craig AR, Franklin JA, Andrews G. A scale to measure locus of control of behaviour. $\mathrm{Br}$ J Med Psychol 1984;57:173-80.

21 Robertson P, Beynon S, Whybin R, Brennan C, Vollmer-Conna U, Hickie I, et al. Measurement of EBV-IgG anti-VCA avidity aids the early and reliable diagnosis of primary EBV infection.J Med Virol 2003;70:617-23.

22 Worswick D, Marmion BP. Antibody responses in acute and chronic $Q$ fever and in subjects vaccinated against $Q$ fever.J Med Microbiol 1985;19:281-96.

23 Rickinson A, Kieff E. Epstein-Barr virus. In: Knipe D, Howley P, eds. Field's virology. Philadelphia, PA: Lippincott, Williams \& Wilkins, 2001:2575-628.

24 Maurin M, Raoult D. Q fever. Clin Microbiol Rev 1999;12:518-53.

25 Harley D, Sleigh A, Ritchie S. Ross River virus transmission, infection and disease: a cross-disciplinary review. Clin Microbiol Rev 2001;14:909-32.

26 White PD, Thomas JM, Amess J, Grover SA, Kangro HO, Clare AW. The existence of a fatigue syndrome after glandular fever. Psychol Med 1995;25:907-16.

27 White PD, Thomas JM, Amess J, Crawford DH, Grover SA, Kangro HO, et al. Incidence, risk and prognosis of acute and chronic fatigue syndromes and psychiatric disorders after glandular fever. Br J Psychiatry 1998;173:475-81.

28 White PD, Thomas JM, Kangro HO, Bruce-Jones WD, Amess J, Crawford DH, et al. Predictions and associations of fatigue syndromes and mood disorders that occur after infectious mononucleosis. Lancet 2001;358:1946-54.

29 Buchwald DS, Rea T, Katon W, Russo JE, Ashley RL. Acute infectious mononucleosis characteristics of patients who report failure to recover. Am J Med 2000;109:531-7.

30 Candy B, Chalder T, Cleare AJ, Peakman A, Skowera A, Wessely S, et al. Predictors of fatigue following the onset of infectious mononucleosis. Psychol Med 2003;33:847-55.

31 White PD, Thomas JM, Sullivan PF, Buchwald D. The nosology of sub-acute and chronic fatigue syndromes that follow infectious mononucleosis. Psychol Med 2004:34:499-507.

32 Wilson A, Hickie I, Hadzi-Pavlovic D, Wakefield D, Parker G, Straus SE, et al. What is chronic fatigue syndrome? Heterogeneity within an international multicentre study. Aust NZJ Psych 2001;35:520-7.

33 Vollmer-Conna U, Fazou C, Cameron B, Li H, Brennan C, Luck L, et al. Production of pro-inflammatory cytokines correlates with the symptoms of acute sickness behaviour in humans. Psychol Med 2004;34:1289-97.

34 Vollmer-Conna U. Acute sickness behaviour: an immune system-to-brain communication? Psychol Med 2001;31:761-7.

35 Cameron B, Bharadwaj M, Burrows J, Fazou C, Wakefield D, Hickie I, et al. Prolonged illness following infectious mononucleosis is associated with altered immunity but not elevated viral load. J Infect Dis 2006;193:664-71.

(Accepted 2 August 2006)

doi 10.1136/bmj.38933.585764.AE

Brain and Mind Research Institute, Sydney University, Sydney, NSW 2050, Australia

Ian Hickie psychiatrist

Tracey Davenport biostatistician

School of Medical Sciences, University of New South Wales, Sydney, NSW 2052

Denis Wakefield immunologist

Barbara Cameron research fellow

Andrew Lloyd infectious diseases physician

School of Psychiatry, University of New South Wales

Ute Vollmer-Conna psychologist

Division of Viral and Rickettsial Diseases, Centers for Disease Control and

Prevention, Atlanta, GA 31033, USA

Suzanne D Vernon molecular virologist

William C Reeves epidemiologist

Correspondence to: A Lloyd a.lloyd@unsw.edu.au 